\title{
Wearing an Anti-COVID Face Mask Predisposes to Spontaneity and Ideas' Expression in Social Interactions: Findings from a Pilot Experiment
}

\author{
Matteo Perini ${ }^{1} \cdot$ Simona Sciara ${ }^{2}$ (D)
}

Accepted: 16 December 2021/Published online: 3 January 2022

(C) Associação Brasileira de Psicologia 2021

\begin{abstract}
Can wearing an anti-COVID face mask bring any positive effects on social interactions? Based on objective self-awareness theory (OSA), our pilot experiment tested whether wearing an anti-COVID-19 face mask-a facial covering that should reduce self-focused attention-can predispose people to spontaneity in social interactions. Upon randomization $(N=91)$, participants were asked to either wear or not wear a mask while completing an online survey that assessed their willingness to be spontaneous in various imagined social situations (e.g., willingness to express ideas in public). As expected, participants who completed the survey while wearing a face mask reported higher levels of anticipated spontaneity, declaring they would have been more willing to express their ideas in the proposed interactions than those who did not wear a mask. Results support the hypothesis that anti-COVID face masks reduce objective self-awareness by drawing others' focus away from the person's face. This effect seems to eventually encourage people's propensity to spontaneity and ideas' expression in social interactions. Implications concerning both positive and negative potential consequences of this effect, as well as possible directions for deepening the study of social effects of anti-COVID measures and further testing the theory of objective self-awareness, are discussed.
\end{abstract}

Keywords Face mask $\cdot$ Objective self-awareness $\cdot$ Spontaneity $\cdot$ Social interaction $\cdot$ COVID-19 - Coronavirus

Simona Sciara

simona.sciara@outlook.com

Matteo Perini

matteoperini.ph@gmail.com

1 Erasmus University Rotterdam, Burgemeester Oudlaan, 50, 3062 Rotterdam, PA, Netherlands

2 Department of Psychology, Catholic University of the Sacred Heart, Largo Agostino Gemelli, 1, 20123 Milano, MI, Italy 
Can wearing an anti-COVID face mask bring any positive effect on social interactions? In an era where wearing a mask is essential to counteract the spread of the coronavirus disease 2019 (COVID-19; e.g., Eikenberry et al., 2020; Leung et al., 2020), several studies have shown that surgical face masks can compromise interpersonal communication (e.g., Bandaru et al., 2020; Carbon, 2020; Karagkouni, 2021). Yet, the potential positive effects of anti-COVID masks on their wearers' social attitudes remain unexplored. The present work aims to apply past theoretical frameworks to fix this gap and test whether a medical mask used to limit the spread of COVID-19 can have positive effects on social interactions. Specifically, as it covers a portion of the face reducing self-focused attention, we suggest that wearing an anti-COVID-19 face mask increases people's spontaneity in social interactions.

In general, the psychological effects of wearing a mask have already been proposed and reported in the past (Castle, 1986; Gell, 1975; MacGowan \& Rosse, 1924). Facial masks, defined as coverings for all or part of the face (e.g., theater masks, makeup, and costumes), have been associated with disinhibition, transformation, facilitation of self-expression, and other psycho-somatic changes (Cooper, 1999). To our best knowledge, however, no study has ever generalized these effects to anti-COVID-19 face masks (e.g., surgical masks, FFP2 masks, and N95 respirators). In other words, there is no proof that anti-COVID face masks can bring similar effects for their wearers.

On the other hand, inferring that medical face masks bring the same effects as other non-medical masks is not a straightforward move. First, one of the most cited explanations for the disinhibiting effects of facial masks relies on the anonymity one can achieve by covering their face. People wearing masks indeed feel less identifiable and thus experience more disinhibition (e.g., Emunah, 1994; Saigre, 1989; Baptiste, 1989), but anti-COVID face masks do not reach the same coverage of integral facial masks, and thus do not ensure complete anonymity. Secondly, the specific meaning of medical masks could interfere with the effects that other (non-medical) masks usually have on their wearers. Indeed, a variety of studies have shown that clothes affect both cognition and behavior in virtue of what they symbolize (Adam \& Galinsky, 2012; Civile \& Obhi, 2017; Karl et al., 2013; Mendoza \& Parks-Stamm, 2020; Slepian et al., 2015), and medical face masks should convey meanings related to the pandemic, such as social distancing measures. Then, why should one expect that anti-COVID face masks can improve spontaneity in social interactions?

Previous research on facial masks' psychological effects (e.g., Cooper, 1999; Johnson \& Downing, 1979) proposed that the disinhibiting effects of wearing a mask can also be explained in terms of a reduced degree of self-awareness. The theory of objective self-awareness (OSA; Duval \& Wicklund, 1972; Silvia \& Duval, 2001; Wicklund, 1975) defines "objective self-awareness" as a psychological state in which the individual orients their attention towards themself, thus taking the self as an object. This self-focused attention makes the person recognize the potential distance that may exist between their actual self and ideal self-a discrepancy between how the person currently is (i.e., actual attainment) and the standards they strive to achieve in terms of "correct" behavior, attitudes, and traits (i.e., aspirations). Since this discrepancy is usually negative, OSA often coincides with an aversive affective drive that motivates the person to reduce the discrepancy, for instance by changing 
the self to match standards (e.g., trying to speak like a sociable guy, or behaving like a good student). This drive can thus interfere with spontaneity in social interactions, with the person trying to control their behavior to adhere to the standards (e.g., a person can inhibit their expression of ideas if these may disappoint the interlocutor).

The attentional focus of the individual changes its orientation from outward to inward depending on various stimuli that remind the person of their objective status (e.g., Wicklund \& Duval, 1971; Ickes et al., 1973). Factors that increase selfawareness include occasions for social comparison, evaluative judgments, mirrors, audiotapes of the person's voice, and any other symbols or reflection of the self that is capable of inducing self-observation, such as the knowledge of being attended by others. In this latest case, the presence of an audience does not automatically induce self-awareness, but the self readily becomes the focus of attention once the person realizes that the audience is paying attention right to some of their personal features or if failures and negative judgments become probable (e.g., in public speaking; Wicklund, 1975; when going public on social media; Sciara et al., 2021).

On the other hand, a series of stimuli can reduce self-awareness by drawing the individual's attention outward, away from the self. These stimuli mainly include distractors, such as a television program, a concert, or a distracting noise, but also active motor actions (e.g., physical activities and sports; Duval \& Wicklund, 1973), and any other factor that is capable of deviating attention away from the intra-self discrepancies, such as deindividuation (e.g., being in a group of masked, interchangeable people; Duval \& Wicklund, 1972; Silvia \& Duval, 2001). More than that, because of the motivation generated by the perception of a discrepancy within the self, the person is also expected to actively avoid those stimuli that induce selffocused attention. This will result in an aversion to mirrors, the focus of others, cameras, and other comparable stimuli (Wicklund, 1975). As a derivation, since anti-COVID-19 masks cover the person's appearance and draw others' focus away from their face, wearing them should reduce self-focused attention and objective self-awareness, finally encouraging more spontaneous interactions with others. This effect should be even more evident in specific social situations in which the attention of others on the person's self is greater, such as in public, or in the company of people who one is not familiar with or does not want to disappoint.

Other frameworks and models also support the above prediction. According to Carver and Scheier's control theory (1981), a reduced self-attention should decrease the inhibitory effect of behavioral standards on actual behavior, with masked people behaving more spontaneously (e.g., Scheier et al., 1974). More generally, face masks provide a sense of protection (Saigre, 1989), which is expected to be even reinforced in the case of anti-COVID face masks, since they actually protect the individual from getting infected by coronavirus (e.g., Nakayachi et al., 2020). Similarly, according to embodied cognition theorists (e.g., Shapiro, 2019), the physical features of the agent's body, including a material covering of the face, influence cognitive processes. In this sense, wearing an anti-COVID mask should reduce the person's perceived vulnerability and in turn elicit a more spontaneous attitude in social interactions.

Based on OSA theory and the above conceptualizations, the present pilot experiment tested whether wearing an anti-COVID-19 face mask induces people to feel 
Table 1 Participants' spontaneity in social interactions depending on whether they were wearing an antiCOVID-19 face mask or not

\begin{tabular}{|c|c|c|c|c|}
\hline & \multicolumn{2}{|c|}{ Face mask wearing } & \multirow[t]{2}{*}{$t$} & \multirow[t]{2}{*}{$p$} \\
\hline & No mask & Mask up & & \\
\hline$M(S D)$ & $2.00(.64)$ & $2.42(.81)$ & 2.61 & .012 \\
\hline$M d n$ & 2.00 & 2.27 & & \\
\hline Bootstrap 95\% CI & {$[1.85,2.17]$} & {$[2.16,2.69]$} & & \\
\hline$N$ & 57 & 34 & & \\
\hline
\end{tabular}

Note. Means, standard deviations, and medians refer to participants' spontaneity in various imagined social interactions (11 items, e.g., willingness to express sincere ideas in public). Scales ranged from 1 "not at all" to 5"very much." Bootstrap estimates for 95\% CIs for the means were obtained with 5,000 resamples. $N$ s indicate the numerosity of each experimental group

more spontaneous and thus more willing to express their ideas in various social interactions. For the purpose, we referred to and operationalized a specific definition of the concept of spontaneity that directly stems from the OSA theory. Spontaneity can be seen as a condition in which the person is free from the constraints due to the state of objective self-awareness-a condition in which their attentional focus would have been oriented towards themself and that could produce anxiety and embarrassment, compromising concentration and ideas' expression (e.g., Duval \& Wicklund, 1972; Silvia \& Duval, 2001; Wicklund, 1975). The study applied an experimental design in which participants were asked to either wear or not wear a mask while completing an online survey ${ }^{1}$ that assessed their expected spontaneity in several imagined social situations, both in terms of expected feelings and behavior. In this regard, it is important to note that imagined events can evoke similar feelings to those induced by perceived events (e.g., Suengas \& Johnson, 1988) and that intentions can be treated - at least in pilot tests—as a good proxy of behavior (e.g., see the theory of planned behavior; Ajzen, 1985).

\section{Method}

\section{Participants, Design, and Sensitivity Power Analysis}

Ninety-one Italian adults $\left(67.0 \%\right.$ females; $M_{\text {age }}=22.26$ years, $\left.S D_{\text {age }}=3.94\right)$ volunteered in a one-factorial between-subject experiment in which the wearing of an anti-COVID-19 face mask was manipulated (Manipulation of Face Mask Wearing: no mask vs. mask up). ${ }^{2}$ Participants' spontaneity in social interactions (e.g., their

\footnotetext{
1 Data collection was conducted during the COVID-19 pandemic with social distancing measures in place.

2 Notably, participants randomly assigned to conditions did not complete the online questionnaire in equal proportions (i.e., 57 in the "no mask" condition, and 34 in the "mask up" condition). The sensitivity power analysis was thus computed with actual numbers reached in the randomized conditions (see Table 1).
} 
willingness to express their ideas in different social situations) served as the dependent variable. The study had $80 \%$ power to detect an effect size of at least $d=0.54$ in pairwise comparisons (i.e., a medium effect according to Cohen, 1988; $\alpha=0.05$; one-tailed tests; non-centrality parameter $\delta=2.51$; see $\mathrm{G}^{*}$ Power 3.1, Faul et al., 2007).

\section{Procedure and Materials}

The research was introduced to participants as investigating people's sensations and opinions concerning the wearing of anti-COVID face masks. Before starting, a brief text warned participants that during completion, the survey might require them to wear an anti-COVID mask or not, and that we needed them to carefully follow instructions in both cases. Hence, participants that at that moment could not guarantee that they could follow the instructions-i.e., that already knew they could not decide to wear or not wear a mask-were kindly invited to leave the survey before starting completion and, if they wanted, resume it later.

After participants gave their informed consent, they filled out an anonymous online questionnaire. The questionnaire consisted of two parts. The first part entailed demographic questions and the instruction concerning the manipulation. Upon randomization, participants in the "mask up" condition were asked to wear a mask for the entire duration of the survey, while participants in the "no mask" condition were asked not to wear a mask (i.e., "For the completion of this questionnaire, you are required to wear/not to wear an anti-COVID face mask, covering nose and mouth").

The second part of the questionnaire consisted of 11 items intended to measure participants' spontaneity in various social interactions while wearing an antiCOVID face mask (dependent variable). Each item was ad hoc created and incorporated (a) a short description of a social situation in which certain elements of the interaction would elicit objective self-awareness ${ }^{3}$ (e.g., having to answer a difficult question from the professor in front of the entire class) and (b) a specific question intended to evaluate participants' expected spontaneity in the presented situation. For example, participants answered the following questions: "When wearing a mask, do you feel comfortable and inclined to interact with a group of people you are not familiar with?"; "Could wearing a mask make it easier for you to express a sincere idea which might raise the disappointment of your interlocutor?"; "In a new class in which you do not know anyone, does wearing a mask make you feel more willing to take the first step and introduce yourself first?"; "During a seminar, a question for the speaker that could be naive or trivial comes to your mind. Could wearing a mask make you feel more comfortable with making your question?"; "Imagine there is a person in front of you that you like. Would wearing a face mask make it easier for you to ask them for a date?". Notably, although the dependent variable's questionnaire referred to spontaneity "while wearing a mask," only participants in the "mask up" condition actually wore a mask in completing the survey. We thus

\footnotetext{
${ }^{3}$ In the questionnaire measuring spontaneity in social interactions, some of the items focused on participants' subjective experiences associated with objective self-awareness (e.g., feeling observed or judged by others), while other items focused on their willingness to spontaneously express ideas and thoughts in specific situations (e.g., raising questions in public or complaining aloud about something).
} 
maintained the control and the experimental conditions (no mask vs. mask up) as similar as possible - the only difference between the two regarded the actual wearing of a mask, the facial barrier expected to reduce objective self-awareness. In so doing, we reduced the plausibility of several alternative explanations, including a priming explanation ${ }^{4}$ (e.g., Doyen et al., 2014; Klauer, 1997; Weingarten et al., 2016). Answers were provided on Likert-like scales ranging from 1 (not at all) to 5 (very тисh). The mean of the 11 items indicated participants' total anticipated spontaneity in social interactions while wearing a mask. The reliability of the scale was adequately high (Cronbach's $\alpha=0.85 ; 11$ items). At the end of the survey, participants were asked to confirm they wore/did not actually wear a mask, depending on their experimental condition. All participants followed instructions. Finally, they were debriefed and thanked for their participation.

\section{Results}

Table 1 summarizes the descriptives and the main results of the study. As expected, participants who completed the survey while wearing a face mask reported higher levels of spontaneity in social interactions (e.g., more willingness to express their ideas) than those who did not wear a mask, $t(57)=2.61, p=0.012, d=0.59$. Levene's test indicated unequal variances, $F=6.25, p=0.014$, so the $t$ test's degrees of freedom were adjusted from 89 to 57 . In addition, considering the non-equality of variances among the two experimental groups, we sought confirmation of this result through a non-parametric test. A Mann-Whitney test confirmed the hypothesized effect, with participants in the "mask up" condition reporting a higher spontaneity than their counterparts, $U=1,263.50, p=0.016$.

\section{Discussion}

Drawing on OSA theory (Duval \& Wicklund, 1972), we hypothesized and found that anti-COVID-19 face masks increase their wearers' willingness to be spontaneous in social interactions, for example, by encouraging the expression of ideas in demanding social circumstances (e.g., in public). This result supports the idea that medical masks reduce objective self-awareness by drawing the person's attentional focus away from the self and that this shift consequently increases social spontaneity.

\footnotetext{
4 According to a priming explanation, wearing anti-COVID masks can induce the psychological recalling and activation of other concepts related to those masks - in our case, for example, the concept of protection, because masks protect from the virus and counter the pandemic, or the concept of social approval, since, during a pandemic, wearing masks is positively viewed from others. This kind of thoughts and respective affective evaluations, however, would have qualified as an alternative explanation for the reported effect on spontaneity in social interactions. We tried to control for this and similar alternative explanations by making participants in the "no mask" and "mask up" groups imagine themselves in the same identical situations-e.g., expressing ideas in public while wearing a mask-where only the experimental group actually wore an anti-COVID mask (i.e., a facial barrier).
} 
According to other consistent conceptualizations, however, the disinhibitory effect of anti-COVID face masks could also derive from a sense of psychological protection due to using medical devices (Nakayachi et al., 2020). Further studies are welcome to elucidate which specific process actually mediated the present result, possibly isolating the protective effect from the covering effect induced by wearing masks. As we explained, only the latter is expected to reduce objective self-awareness. For instance, if the present effect of masks on spontaneity is explained by OSA, we should expect it to disappear in the case of transparent masks, which ensure protection without covering the lower portion of the face (Marini et al., 2021). Conversely, if the increased spontaneity in social interactions is due to the sense of protection, face masks should affect social behavior in the same way, regardless of whether they are transparent or not. In this sense, the theory-driven inclusion of pertinent mediators and/or moderators in future studies will certainly shed light on this and possibly extend the evidence in favor of the proposed process.

Another alternative explanation that should be considered in interpreting our result is the possibility that the reported effect was caused-or co-caused-by a meaning-making process with respect to the task of wearing a mask while completing a computer assignment. The requirement to wear a mask while doing something on a computer might have sounded strange to our participants and thus have stimulated, in them, attempts at making meaning for the proposed procedure. However, even if we cannot exclude that a process of meaning making interfered with the current experimental test, it is important to consider that, before the survey, participants in both the groups had been warned that, during completion, the computer might have required them to wear an anti-COVID mask or refrain from doing so. Participants who could not follow the instructions were thus invited to leave the survey or, if they wanted, complete it later. This means that a potential process of meaning making-if it occurred-plausibly involved both groups, thus reducing the difference between conditions and, in turn, the probability that our result was due to meaning making rather than to wearing an anti-COVID mask. In any case, to exclude this alternative explanation, future scholars might want to introduce in their research procedures a new experimental condition in which participants are requested to do something strange while completing the survey-i.e., something that is equivalent to wearing a mask but not implying facial coverings (e.g., the requirement to wear a jacket).

Other limitations of the present study may concern the nature of our measurements and the recruited sample. The questionnaire we used was indeed highly reliant on self-reports, including the item intended to control that our participants followed the survey's instructions. Also, we only examined participants' expected levels of spontaneity and intentions to interact with others in a series of imagined situations, and not their actual feelings, or social behavior (e.g., we measured their willingness to express ideas in public, not their actual expression of ideas). Then, even if imagined events can induce similar feelings to those induced by perceived events (e.g., Suengas \& Johnson, 1988) and intentions might be a good predictor of behavior (e.g., see the theory of planned behavior; Ajzen, 1985), future studies should confirm the presented finding by implementing behavioral measurements of spontaneity and measuring respective feelings contextually. Another limitation 
stems from the demographic characteristics of our sample (i.e., mainly young and female), which are associated with a stronger influence of physical appearance on self-esteem (Pliner et al., 1990). This influence, in turn, may have increased selfefficacy and self-confidence in the "mask up" condition (e.g., Boyd \& Hrycaiko, 1997), thus representing an alternative explanation for the reported result. Similarly, other individual differences might have intervened in producing the observed effect and should thus be considered as control variables by future research.

Despite its limitations, our study suggests that wearing an anti-COVID mask can improve some aspects of social communication. We found first evidence that facial masks can bring social benefits for their wearers by orienting their attentional focus away from the self, and thus facilitating their social interactions and a more spontaneous expression of ideas. With a mask, in fact, the individual's attempts to control their expression of thoughts to meet standards would be less intense, with their attentional focus orienting outward, towards the social exchange. Consequently, being free from the constraints due to objective self-awareness might finally lead to several benefits. A reduced OSA could for example help the person feel less anxious, comment aloud, express their ideas sincerely, concentrate on what others are saying, and thus promote a natural social exchange and a better comprehension, all critical aspects of communication. Furthermore, as suggested by previous scholars, spontaneity is generally associated with a number of other positive psychosocial outcomes, such as increased emotions recognition, creativity, and learning (e.g., Kipper et al., 2010; Mangalam \& Guha, 2017; Ramdani \& Rahmat, 2018). In this sense, our result not only encourages the study of the positive effects of wearing a mask but also the exploration of the potential social benefits of other anti-COVID recommendations/ restrictions, such as the benefits of home isolation on families' time spent together and consequent levels of happiness (Güzel et al., 2020).

As demonstrated so far, however, face masks can also have negative effects on social relationships, such as a reduced emotional connection between people (Carbon, 2020). Similarly, the effect of anti-COVID masks on spontaneous social interactions should be considered more critically. Appropriate interaction is the best interaction possible, and, in some situations, spontaneity may not represent the most appropriate way to interact with others-e.g., if it might offend or disappoint people. In its extreme manifestations, spontaneity could even lead to impulsiveness and disinhibition, translating into tactless, rude, or even offensive actions. This would finally affect how people comply with rules and social norms. A decrement of OSA may thus reverberate in the moral domain and increase the perception of anonymity and deindividualization, with a higher subsequent risk of engaging in antisocial behaviors (e.g., Zimbardo, 1969).

\section{Conclusions}

Drawing on the theory of OSA (Duval \& Wicklund, 1972), we found that wearing anti-COVID-19 face masks increases people's willingness to be spontaneous in social interactions. This finding supports OSA theory by suggesting that, since masks cover a substantial portion of the face, wearing them reduces objective 
self-awareness and encourages spontaneity in social interactions. In guiding future research, this first result not only represents an initial contribution to fixing the literature gap on the positive social effects of wearing masks but also permits a better understanding of broader psychosocial processes. Wearing anti-COVID-19 masks seems to facilitate some aspects of social communication by increasing spontaneity and avoiding constraints associated with OSA. Spontaneity, however, can either be positive or negative for social interactions. Inappropriate spontaneity, for instance, can induce a feeling of deindividualization and in turn a higher risk of antisocial behaviors. In this sense, our pilot study highlights the need to move beyond a conception of the face mask as a mere protective device but as an item with psychosocial implications and cultural significance.

Abbreviations COVID: Coronavirus disease; COVID-19: Coronavirus disease 2019; OSA: Objective self-awareness

Acknowledgements The authors would like to greatly acknowledge Francesca Falbo and Sofia Colombo for their help and contribution in the collection of data and the development of the questionnaire used in this study.

Availability of Data and Material The authors are willing to share their data, analytics methods, and study materials with other researchers upon request.

Code Availability Not applicable since the authors used basic SPSS functions for their statistical analyses.

\section{Declarations}

Ethical Approval All procedures performed in the study were in accordance with APA ethical guidelines and the 1964 Helsinki declaration and its later amendments.

Informed Consent to Participate All participants gave full informed consent to participate.

Consent for Publication All participants gave consent for their data to be used in publication.

Conflict of Interest The authors declare no competing interests.

\section{References}

Adam, H., \& Galinsky, A. D. (2012). Enclothed cognition. Journal of Experimental Social Psychology, 48(4), 918-925. https://doi.org/10.1016/j.jesp.2012.02.008

Ajzen, I. (1985). From intentions to actions: A theory of planned behavior. In: Kuhl J., Beckmann J. (eds) Action control. SSSP Springer Series in Social Psychology. Springer: Berlin, Heidelberg. https://doi. org/10.1007/978-3-642-69746-3_2

Bandaru, S. V., Augustine, A. M., Lepcha, A., Sebastian, S., Gowri, M., Philip, A., \& Mammen, M. D. (2020). The effects of N95 mask and face shield on speech perception among healthcare workers in the coronavirus disease 2019 pandemic scenario. The Journal of Laryngology \& Otology, 134, 895-898. https://doi.org/10.1017/S0022215120002108

Boyd, K. R., \& Hrycaiko, D. W. (1997). The effect of a physical activity intervention package on the selfesteem of pre-adolescent and adolescent females. Adolescence, 32(127), 693-708.

Carbon, C. C. (2020). Wearing face masks strongly confuses counterparts in reading emotions. Frontiers in Psychology, 11, 566886. https://doi.org/10.3389/fpsyg.2020.566886

Carver, C. S., \& Scheier, M. F. (1981). Attention and self-regulation. Springer-Verlag. 
Castle, T. (1986). Masquerade and civilization. Methuen.

Civile, C., \& Obhi, S. S. (2017). Students wearing police uniforms exhibit biased attention toward individuals wearing hoodies. Frontiers in Psychology, 8, 62. https://doi.org/10.3389/fpsyg.2017.00062

Cohen, J. (1988). Statistical power analysis for the behavioral sciences. Lawrence Erlbaum Associates.

Cooper, M. (1999). An empirical and theoretical investigation into the psychological effects of wearing a mask [Doctoral dissertation, University of Sussex]. Strathprints. Retrieved from https://strathprints. strath.ac.uk/43402/

Doyen, S., Klein, O., Simons, D. J., \& Cleeremans, A. (2014). On the other side of the mirror: Priming in cognitive and social psychology. Social Cognition, 32, 12-32. https://doi.org/10.1521/soco.2014. 32.supp. 12

Duval, S., \& Wicklund, R. A. (1972). A theory of objective self-awareness. Academic Press.

Duval, S., \& Wicklund, R. A. (1973). Effects of objective self-awareness on attribution of causality. Journal of Experimental Social Psychology, 9(1), 17-31. https://doi.org/10.1016/0022-1031(73) 90059-0.

Eikenberry, S. E., Mancuso, M., Iboi, E., Phan, T., Eikenberry, K., Kuang, Y., et al. (2020). To mask or not to mask: Modeling the potential for face mask use by the general public to curtail the COVID19 pandemic. Infectious Disease Modelling, 5, 293-308. https://doi.org/10.1016/j.idm.2020.04.001

Faul, F., Erdfelder, E., Lang, A.-G., \& Buchner, A. (2007). G*Power 3: A flexible statistical power analysis program for the social, behavioral, and biomedical sciences. Behavior Research Methods, 39(2), 175-191. https://doi.org/10.3758/BF03193146

Gell, A. (1975). Metamorphosis of the Cassowaries. Athlone Press.

Güzel, P., Yıldız, K., Esentaş, M., \& Zerengök, D. (2020). "Know-how" to spend time in home isolation during COVID-19; restrictions and recreational activities. International Journal of Psychology and Educational Studies, 7(2), 122-131. https://doi.org/10.17220/ijpes.2020.02.011

Ickes, W. J., Wicklund, R. A., \& Ferris, C. B. (1973). Objective self awareness and self esteem. Journal of Experimental Social Psychology, 9(3), 202-219. https://doi.org/10.1016/0022-1031(73)90010-3.

Johnson, R. D., \& Downing, L. L. (1979). Deindividuation and valence of cues: Effects on prosocial and antisocial behavior. Journal of Personality and Social Psychology, 37(9), 1532-1538. https://doi. org/10.1037/0022-3514.37.9.1532

Karagkouni, O. (2021). The effects of the use of protective face mask on the voice and its relation to selfperceived voice changes. Journal of Voice. https://doi.org/10.1016/j.jvoice.2021.04.014

Karl, K. A., Hall, L. M., \& Peluchette, J. V. (2013). City employee perceptions of the impact of dress and appearance: You are what you wear. Public Personnel Management, 42(3), 452-470. https://doi.org/ $10.1177 / 0091026013495772$

Kipper, D. A., Green, D. J., \& Prorak, A. (2010). The relationship among spontaneity, impulsivity, and creativity. Journal of Creativity in Mental Health, 5(1), 39-53. https://doi.org/10.1080/1540138100 3640866

Klauer, K. C. (1997). Affective priming. European Review of Social Psychology, 8(1), 67-103. https:// doi.org/10.1080/14792779643000083

Leung, N. H. L., Chu, D. K. W., Shiu, E. Y. C., et al. (2020). Respiratory virus shedding in exhaled breath and efficacy of face masks. Nature Medicine, 26, 676-680. https://doi.org/10.1038/ s41591-020-0843-2

MacGowan, K. \& Rosse, H. (1924). Masks and demons. Martin Hopkinson \& Co.

Mangalam, K., \& Guha, T. (2017). Learning spontaneity to improve emotion recognition in speech. arXiv preprint, 1712.04753. https://arxiv.org/pdf/1712.04753.pdf

Marini, M., Ansani, A., Paglieri, F., Caruana, F., \& Viola, M. (2021). The impact of facemasks on emotion recognition, trust attribution and re-identification. Scientific Reports, 11, 5577. https://doi.org/ 10.1038/s41598-021-84806-5

Mendoza, S. A., \& Parks-Stamm, E. J. (2020). Embodying the police: The effects of enclothed cognition on shooting decisions. Psychological Reports, 123(6), 2353-2371. https://doi.org/10.1177/00332 94119860261

Nakayachi, K., Ozaki, T., Shibata, Y., \& Yokoi, R. (2020). Why do Japanese people use masks against COVID-19, even though masks are unlikely to offer protection from infection? Frontiers in Psychology, 11, 1918. https://doi.org/10.3389/fpsyg.2020.01918

Pliner, P., Chaiken, S., \& Flett, G. L. (1990). Gender differences in concern with body weight and physical appearance over the life span. Personality and Social Psychology Bulletin, 16(2), 263-273. https://doi.org/10.1177/0146167290162007 
Ramdani, J. M., \& Rahmat, R. (2018). Promoting speaking spontaneity in large classes: An action research study in an Indonesian EFL university setting. Indonesian Journal of Applied Linguistics, 8(2), 388-401. https://doi.org/10.17509/ijal.v8i2.13304

Saigre, H. (1989). La mascothérapie: Une psychothérapie post-Reichienne. L'information Psychiatrique, 10, 1017-1023.

Scheier, M. F., Fenigstein, A., \& Buss, A. H. (1974). Self-awareness and physical aggression. Journal of Experimental Social Psychology, 10(3), 264-273. https://doi.org/10.1016/0022-1031(74)90072-9

Sciara, S., Contu, F., Bianchini, M., Chiocchi, M., \& Sonnewald, G. G. (2021). Going public on social media: The effects of thousands of Instagram followers on users with a high need for social approval. Current Psychology. https://doi.org/10.1007/s12144-021-02172-x.

Shapiro, L. (2019). Embodied cognition (2nd ed.). Routledge.

Silvia, P. J., \& Duval, T. S. (2001). Objective self-awareness theory: Recent progress and enduring problems. Personality and Social Psychology Review, 5(3), 230-241. https://doi.org/10.1207/S1532 7957PSPR0503_4

Slepian, M. L., Ferber, S. N., Gold, J. M., \& Rutchick, A. M. (2015). The cognitive consequences of formal clothing. Social Psychological and Personality Science, 6(6), 661-668. https://doi.org/10.1177/ 1948550615579462

Suengas, A. G., \& Johnson, M. K. (1988). Qualitative effects of rehearsal on memories for perceived and imagined complex events. Journal of Experimental Psychology: General, 117(4), 377-389. https:// doi.org/10.1037/0096-3445.117.4.377

Weingarten, E., Chen, Q., McAdams, M., Yi, J., Hepler, J., \& Albarracin, D. (2016). On priming action: Conclusions from a meta-analysis of the behavioral effects of incidentally-presented words. Current Opinion in Psychology, 12, 53-57. https://doi.org/10.1016/j.copsyc.2016.04.015

Wicklund, R. A. (1975). Objective self-awareness. Advances in Experimental Social Psychology, 8, 233275. https://doi.org/10.1016/S0065-2601(08)60252-X

Wicklund, R. A., \& Duval, S. (1971). Opinion change and performance facilitation as a result of objective self-awareness. Journal of Experimental Social Psychology, 7(3), 319-342. https://doi.org/10.1016/ 0022-1031(71)90032-1.

Zimbardo, P. G. (1969). The human choice: Individuation, reason, and order versus deindividuation, impulse, and chaos. Nebraska Symposium on Motivation, 17, 237-307. 